\title{
Efficient and Tunable Electroluminescence from In Situ Synthesized Perovskite Quantum Dots
}

Hongling Yu, Heyong Wang, J iangbin Zhang, J un Lu, Zhongcheng Yuan, Weidong $\mathrm{Xu}$, Lars Hultman, Artem A. Bakulin, Richard H. Friend, J ianpu Wang, Xiaoke Liu and Feng Gao

The self-archived postprint version of this journal article is available at Linköping University Institutional Repository (DiVA):

http:// urn.kb.se/ resolve?urn=urn:nbn:se:liu:diva-154995

N.B.: When citing this work, cite the original publication.

Yu, H., Wang, H., Zhang, J., Lu, J ., Yuan, Z., Xu, W., Hultman, L., Bakulin, A. A., Friend, R. H., Wang, J., Liu, X., Gao, F., (2019), Efficient and Tunable Electroluminescence from In Situ Synthesized

Perovskite Quantum Dots, Small, 15(8), 1804947. https:// doi.org/ 10.1002/smll.201804947

Original publication available at:

https:// doi.org/ 10.1002/ smll.201804947

Copyright: Wiley (12 months)

http:// eu.wiley.com/WileyCDA/ 


\section{Efficient and Tunable Electroluminescence from In-situ Synthesized Perovskite Quantum Dots}

Hongling Yu, Heyong Wang, Jiangbin Zhang, Jun Lu, Zhongcheng Yuan, Weidong Xu, Lars Hultman, Artem A. Bakulin, Richard H. Friend, Jianpu Wang, Xiao-Ke Liu* and Feng Gao*

H. Yu, H. Wang, Dr. J. Lu, Z. Yuan, Dr. W. Xu, Prof. L. Hultman, Dr. X.-K. Liu, Dr. F. Gao Department of Physics, Chemistry and Biology (IFM), Linköping University, Linköping 58183, Sweden

E-mail: xiaoke.liu@liu.se; feng.gao@liu.se

J. Zhang, Prof. R. H. Friend, Dr. X.-K. Liu

Cavendish Laboratory, University of Cambridge, J. J. Thomson Avenue, Cambridge CB3 OHE, United Kingdom

Dr. W. Xu, Prof. J. Wang

Key Laboratory of Flexible Electronics (KLOFE) \& Institute of Advanced Materials (IAM), Jiangsu National Synergetic Innovation Center for Advanced Materials (SICAM), Nanjing Tech University, 30 South Puzhu Road, Nanjing 211816, China

J. Zhang, Dr. A. A. Bakulin

Department of Chemistry, Imperial College London, London SW7 2AZ, United Kingdom

Keywords: organic-inorganic hybrid perovskite, light-emitting diodes, tunable emission, energy transfer

Semiconductor quantum dots (QDs) are among the most promising next-generation optoelectronic materials. QDs are generally obtained through either epitaxial or colloidal growth and carry the promise for solution-processed high-performance optoelectronic devices such as light-emitting diodes (LEDs), solar cells, etc. Herein, we report a straightforward approach to synthesize perovskite QDs and demonstrate their applications in efficient LEDs. The perovskite QDs with controllable crystal sizes and properties are in-situ synthesized through one-step spin-coating from perovskite precursor solutions followed by thermal annealing. These perovskite QDs feature size-dependent quantum confinement effect (with readily tunable emissions) and radiative monomolecular recombination. Despite the substantial structural inhomogeneity, the in-situ generated perovskite QDs films emit narrow-bandwidth emission and high color stability due to efficient energy transfer between nanostructures that sweeps away the unfavourable disorder effects. Based on these materials, efficient LEDs with external quantum efficiencies up to $11.0 \%$ have been realized. This makes the technologically 
appealing in-situ approach promising for further development of state-of-the-art LED systems and other optoelectronic devices.

One of the most attractive properties of semiconductor quantum dots (QDs) is a remarkable change of optical properties as a function of the crystal size. ${ }^{[1]}$ In principle, their electronic transitions shift to higher energy with decreasing crystal dimensions, well known as the sizedependent quantum confinement effect. This property makes QDs attractive for the applications in optical and electronic devices, such as light-emitting diodes (LEDs) ${ }^{[2]}$, solar cells ${ }^{[3]}$, quantum communications elements ${ }^{[4]}$, and thermoelectrics ${ }^{[5]}$. In general, semiconductor QDs can be obtained through epitaxial $^{[5]}$ or colloidal ${ }^{[2]}$ growth, using vapor phase and chemical reactions, respectively. In contrast to the epitaxial QDs, colloidal QDs are solution-processable semiconductors. Such materials are compatible with light-weight and flexible plastic substrates and carry promise for future low-cost and large-area optoelectronic devices. ${ }^{[6]}$ In particular, colloidal QDs have easily tunable emission, narrow emission bandwidth, and high photoluminescence (PL) efficiency, making them ideal candidates for LEDs. ${ }^{[7]}$ Since the first demonstration in 1994, ${ }^{[8]}$ colloidal QD LEDs have realized major advances, currently reaching external quantum efficiencies (EQEs) over $20 \%$. $^{[9,10]}$

Recently, metal halide perovskites have emerged as promising solution-processable semiconductors, competitive with the best inorganic semiconductors such as silicon and gallium arsenide. ${ }^{[11]}$ They are not only strong light absorbers with excellent semiconducting properties for solar cells, but also promising light-emitting materials with high PL efficiency, narrow and easily tunable emissive line shapes, and wide color gamut. ${ }^{[12]}$ Since the pioneering work on room-temperature perovskite LEDs was reported in $2014,{ }^{[13]}$ considerable progress has been made through elaborate materials and devices design as well as advanced processing techniques. ${ }^{[14-17]}$ As a consequence, a series of efficient perovskite LEDs have been obtained 
with EQEs approaching $20 \% .{ }^{[18-21]}$ In addition to perovskite thin films, colloidal perovskite QDs (PQDs) have also been developed and achieved high-efficiency LEDs. ${ }^{[22-24]}$ For example, Chiba and co-workers demonstrated a series of efficient LEDs with EQEs up to 21.3\% by using colloidal PQDs. ${ }^{[21]}$

In general, PQDs are synthesized through sophisticated chemical processes, and dispersed into nonpolar solvents to form colloidal solutions before being employed in LEDs. ${ }^{[25]}$ Longchain alkyl ligands such as oleic acid and oleylamine are used to suppress aggregation and precipitation of the PQDs. ${ }^{[26]}$ However, the insulating nature of the alkyl chains restrains injection and transport of charge carriers, resulting in low performance and demanding high driving voltages of the LEDs. ${ }^{[27]}$ Additional purification processes are usually required to remove superfluous alkyl ligands to improve the device performance at the expense of stability of the colloidal PQD solutions.

Herein, we report efficient and tunable electroluminescence (EL) achieved by employing in-situ solution-grown PQDs. In contrast to the epitaxial and colloidal QD materials, the in-situ generated PQDs are synthesized through one-step spin-coating from perovskite precursor solutions, followed by thermal annealing. However, similar to the colloidal PQDs, the in-situ grown PQDs show tunable emissive wavelengths by adjusting annealing temperature and time. In-situ generated $\mathrm{FAPbI}_{3}$ PQDs in this study show considerable PL and EL spectral shifts up to $\sim 120 \mathrm{~nm}$ as a function of annealing temperature. Our joint experiments and calculations suggest that the spectral shifts originate from the size-dependent quantum confinement effect. In addition, these in-situ generated PQDs is dominant with radiative monomolecular recombination, leading to high EQEs up to $11.0 \%$ and a maximum radiance of $178 \mathrm{~W} \mathrm{sr}^{-1} \mathrm{~m}^{-2}$ in LEDs. Our work opens up an effective way for developing highly-efficient perovskite LEDs with readily tunable emission spectra. By tailoring the perovskite compositions, this approach 
can also spur new development of efficient perovskite LEDs with highly desired blue and orange colors.

In this study, we choose aromatic ammonium iodide (1-naphthylmethyl ammonium iodide, NMAI) ${ }^{[17]}$ as the ligand to in-situ form PQDs. The FAPbI ${ }_{3}$ PQDs are synthesized by using onestep spin-coating method from precursor solution of formamidinium iodide (FAI), lead iodide $\left(\mathrm{PbI}_{2}\right)$ and NMAI with a molar ratio of 1:1:1, followed by thermal annealing at various temperatures as shown in Figure 1a. X-ray diffraction (XRD) measurements are performed to study crystal structures of these films. As shown in Figure 1b, in addition to the signals from the ITO (indium tin oxide) substrates, reflection peaks at $14.2^{\circ}$ and $28.5^{\circ}$ can be observed and assigned to the (111) and (222) lattice planes of $\alpha$-phase $\mathrm{FAPbI}_{3}$, respectively. ${ }^{[28]}$ The diffraction intensity gradually decreases along with reduced annealing temperature, suggesting lower film crystallinity at lower annealing temperature. This result is consistent with the observations from the scanning electron microscope (SEM) measurements (Figure S1).

Figure 1c shows the diffraction peaks of the (111) plane with normalized intensity. The diffraction spectra widen with decreasing annealing temperature, indicating decreasing crystal size. The average crystal size $(d)$ of the perovskite crystals in these films can be estimated by using the Scherrer equation ${ }^{[29]}$, which is expressed as

$$
d=\frac{K \lambda}{\beta \cos \theta}
$$

where $K$ is a crystallite shape factor, $\lambda$ is the X-ray wavelength (1.5406 $\AA$ ), $\beta$ and $\theta$ are the FWHM (full width at half maximum) in radian and the Bragg angle of the (111) peak (here $\theta$ is $7.1^{\circ}$ ), respectively. As summarized in Table S1, the average crystal sizes of these films are calculated to be $17.9 \mathrm{~nm}\left(100^{\circ} \mathrm{C}\right), 14.8 \mathrm{~nm}\left(90^{\circ} \mathrm{C}\right), 13.1 \mathrm{~nm}\left(80^{\circ} \mathrm{C}\right), 9.5 \mathrm{~nm}\left(70^{\circ} \mathrm{C}\right), 9.0 \mathrm{~nm}$ $\left(60^{\circ} \mathrm{C}\right)$, and $8.3 \mathrm{~nm}\left(50^{\circ} \mathrm{C}\right)$, respectively.

The existence of PQDs in these films is confirmed by transmission electron microscopy (TEM) measurements. Figure S2 shows TEM images of the FAPbI 3 PQD-based films annealed 
at $100{ }^{\circ} \mathrm{C}$ and $70{ }^{\circ} \mathrm{C}$, respectively. Both two films clearly show discretely distributed $\mathrm{FAPbI}_{3}$ crystals and lattice fringes with $d$-spacing of $\sim 6.6 \AA$, which can be indexed to the (111) lattice plane of $\alpha-\mathrm{FAPbI}_{3} \cdot{ }^{[30,31]}$ These observations indicate that this in-situ method results in PQDs with crystal size of few to tens of nanometers.

We note that, in addition to PQDs, the produced films also include different types of nanoconfined structures. Figure 1d compares normalized UV-vis absorption and PL spectra of the PQD-based films prepared at different annealing temperatures. We attribute the absorption peak at $\sim 570 \mathrm{~nm}$ to the excitonic absorption of bilayer perovskite quantum wells. ${ }^{[17]}$ This is confirmed by the XRD patterns of the films annealed at $90^{\circ} \mathrm{C}$ and $100{ }^{\circ} \mathrm{C}$, where a diffraction peak at $3.83^{\circ}$, corresponding to a $d$-spacing of $23.0 \AA$, can be observed. The intensity of excitonic absorption peak of these films decreases gradually at lower annealing temperatures, consistent with their lower crystallinities observed in SEM and XRD. Later in the manuscript we show that the emission properties of the materials are mainly determined by the QDs with low bandgap due to the high band gap of (narrow) quantum wells and efficient energy transfer between different nanostructures.

The absorption and PL spectra of these films show remarkable blue shifts with decreasing annealing temperature. We speculate that the shifts result from the size-dependent quantum confinement effect. As derived from the XRD data and shown in Table S1, the average crystal size of the films decreases with decreasing annealing temperature. At the same time, the absorption onsets and PL spectra show gradual blue shifts (Figure 1d). The bandgap energy $\left(E_{\mathrm{g}}\right)$ of a quantum-confined system (a finite crystal in which propagation of particles is spatially confined in at least one direction) can be expressed by

$$
E_{\mathrm{g}}=E_{\mathrm{g}, \text { bulk }}+\Delta E_{\text {confinement }}(2),
$$


where $E_{\mathrm{g}, \mathrm{bulk}}$ is the bandgap of its bulk crystal in which particles can propagate freely throughout the crystal and $\Delta E_{\text {confinement }}$ is the particle-in-a-box quantum energy denoting the bandgap increase due to quantum confinement effect. ${ }^{[32]}$ The $\Delta E_{\text {confinement }}$ can be expressed as

$$
\Delta E_{\text {confinement }}=\frac{2 \pi^{2} \hbar^{2}}{m_{e} d^{2}}
$$

where $\hbar$ is the reduced Planck constant, $m_{e}$ is the effective mass of the excitons, and $d$ is the average diameter of the crystals. ${ }^{[32,33]}$ Thus, the relation of the bandgaps between the quantumconfined crystal and the bulk crystal is described as

$$
E_{\mathrm{g}}=E_{\mathrm{g}, \mathrm{bulk}}+\frac{2 \pi^{2} \hbar^{2}}{m_{e}} \frac{1}{d^{2}}
$$

As shown in Figure 1e, the relation between the energy bandgaps (determined from the PL peak positions) and the average diameters of the crystals in these films can be well fitted by

$$
E_{\mathrm{g}}=1.52+\frac{24.7}{d^{2}}(\mathrm{eV})
$$

suggesting a bandgap of $1.52 \mathrm{eV}$ for bulk $\mathrm{FAPbI}_{3}$. This value is close to the experimentally reported $1.53 \mathrm{eV} \cdot{ }^{[34]}$ In addition, by comparing Equation 4 and 5, we obtain the effective mass of the excitons in the PQDs which is $\sim 0.061 m_{0}$, where $m_{0}$ is the real mass of a free electron. This value is quite close to the reported of $0.095 m_{0}{ }^{[35]}$

The exciton Bohr radius, which describes the natural delocalization lengths of an exciton in a bulk semiconductor, can be expressed as

$$
r_{B}=\frac{\varepsilon_{r} \hbar^{2}}{m_{e} e^{2}}=\frac{0.0529 \varepsilon_{r} m_{0}}{m_{e}}(\mathrm{~nm})(6),
$$

where $\varepsilon_{r}$ and $e$ are the relative dielectric constant of the bulk crystal and the unit charge, respectively. Given that the effective dielectric constant is approximately 11.4 for $\mathrm{FAPbI}_{3}{ }^{[35]}$ and its exciton effective mass is $0.061 m_{0}$, the exciton Bohr radius of $\mathrm{FAPbI}_{3}$ is estimated to be $9.9 \mathrm{~nm}$ based on Equation 6. The diameters of the nanocrystals in this study (Table S1) are smaller than the exciton Bohr diameter of $19.8 \mathrm{~nm}$ as deceasing annealing temperature. From these results, we assign the blue shifts of the absorption and PL spectra of these films to the 
size-dependent quantum confinement effect (we will further confirm the existence of the quantum confinement effect in the following context).

Interestingly, the PL spectra of prepared films are found to be increasingly broad with decreasing the annealing temperature (Table S1). This observation can be attributed to different size distributions of the PQDs at various temperatures. As indicated in the TEM images in Figure S2, the crystal dimensions of the PQD-based films are not exactly the same. Moreover, the size distribution becomes broader at lower annealing temperatures, as indicated from the broader PL spectra. However, we find that the color purity of PL is stabilized in respect to the material inhomogeneity due to efficient energy transfer between nanostructures. Since smaller crystals and thin quantum wells have larger bandgaps due to the size-dependent quantum confinement effect, energy transfer from the higher-energy crystal sites to the lower-energy sites can occur. We probe such energy funnelling between crystals of different sizes using timecorrelated single photon counting (TCSPC) and transient absorption (TA) spectroscopy. As shown in Figure S3, for the PQD films annealed at low temperatures $\left(50-80{ }^{\circ} \mathrm{C}\right)$, the lowerenergy emissions (e.g. $\sim 745 \mathrm{~nm})$ show a considerably slower decay rate compared with the higher-energy emissions (600-700 nm). More importantly, the lower-energy emissions exhibit obvious rises within $1 \mathrm{~ns}$ after excitation at $407 \mathrm{~nm}$, indicating that these emission species are formed from energy transfer rather than direct excitation of the corresponding crystals. These observations confirm that there is efficient energy transfer in these PQD films. However, PQD size distribution is remarkably wide in the materials formed at low-temperature annealing conditions and this heterogeneity cannot be fully swept out by the energy transfer. As a result, these films show wider PL spectra compared with those annealed at $90{ }^{\circ} \mathrm{C}$ and $100{ }^{\circ} \mathrm{C}$. However, tailoring the energy landscape in these films and controlling the size distribution of the PQDs could narrow their PL spectra. ${ }^{[36,37]}$ 
The energy transfer between materials’ nanostructures is further confirmed by femtosecond TA measurements (Figure 2). TA spectra contain a broad photo-induced absorption response typical for intragap transitions in perovskites ${ }^{[38]}$ superimposed with three photobleaching (PB) features PB1-PB3. Based on the previous work, we attribute PB1 and PB2 to the bandgap renormalisation and stimulated emission of $2 \mathrm{D}$ quantum wells. ${ }^{[17]}$ By comparison with PL spectra PB3 is attributed to the stimulate emission from PQDs. In agreement with TCSPC results, TA spectra show that the quantum wells and high-gap (small-size) PQDs loose excited state population within the first 0.1-1 ns. Also in accordance with size-distribution measurements samples annealed at $100{ }^{\circ} \mathrm{C}$ demonstrate less heterogeneity and faster energy redistribution, likely associated with more resonant coupling between nanostructures. As a result, energy transfer processes sweep away the disorder effects induced by in-situ synthesis and reward the material with relatively narrow PL emission and high color stability.

Electrical properties of these in-situ grown PQDs are investigated by fabricating LEDs with a device structure of ITO/ZnO (30 nm)/PEIE/PQDs (30 nm)/TFB (40 nm)/MoO 3 (7 nm)/Ag (80 nm) (Figure 3a), where PEIE and TFB are polyethylenimine ethoxylated and poly (9,9dioctyl-fluorene-co-N-(4-butylphenyl)diphenylamine, respectively. Figure 3b shows current density-voltage-radiance curves of these devices. The devices based on the PQDs annealed at lower temperatures exhibit relatively lower current densities compared to those with PQDs annealed at higher temperatures. This can be explained by the fact that the PQDs annealed at lower temperatures tend to have lower crystallinity and thus to be less efficient on charge transport. The turn-on voltages $\left(\mathrm{V}_{\text {on }}\right)$ of the PQD-based devices gradually increase with decreasing annealing temperature because of the correspondingly increasing bandgaps. The PQDs annealed at higher temperatures deliver higher radiance and EQEs in devices (Figure 3b and c). In particular, the PQD film annealed at $100{ }^{\circ} \mathrm{C}$ presents a maximum radiance of $178 \mathrm{~W}$ $\mathrm{sr}^{-1} \mathrm{~m}^{-2}$ at $4.0 \mathrm{~V}$, and a maximum EQE of $11.0 \%$ at $2.1 \mathrm{~V}$ (corresponding to a current density 
of $27 \mathrm{~mA} \mathrm{~cm}^{-2}$ ). However, further raising the annealing temperature to $110{ }^{\circ} \mathrm{C}$ or extending the annealing time at $100{ }^{\circ} \mathrm{C}$ to $20-30$ min remarkably reduce the EQEs of the LEDs (Table S2), which can be attributed to the chemical instability of perovskites on $\mathrm{ZnO}$ substrate at high temperatures. ${ }^{[39]}$

Figure 3d shows normalized EL spectra of the devices with considerable blue shifts from $790 \mathrm{~nm}$ to $667 \mathrm{~nm}$ when decreasing annealing temperature. This trend is in good agreement with the observations in PL spectra discussed above. This corroborates the operation of the quantum confinement effect varying from the controllable crystal size by thermal annealing. There are two main strategies for tuning emission colors of perovskite emitters. One is based on size-dependent colloidal PQDs that require sophisticated chemical synthesis processes. ${ }^{[40]}$ The other is using mixed-halogen perovskites, which however, suffer from instability problems due to phase segregation ${ }^{[41]}$ and severe PL quenching ${ }^{[17]}$. Thus, the result of the tunable EL spectra of our devices is of great significance because it demonstrates the fact that various emission colors can be realized in LEDs based on pure-halogen perovskite thin films. These pure-halogen perovskites can be easily obtained through simple deposition process and free of phase segregation with good spectral stability under working conditions (Figure S4 and Figure S5). In addition, the device performance of the emission-tunable devices is acceptable, showing low turn-on voltages of 1.2-2.0 V, EQEs of $0.4-11.0 \%$ and device lifetime up to 142 min (the time duration to 60\% of the initial EQE value) (Table 1, Figure 3 and Figure S6), which may be further improved by device engineering.

Carrier dynamics of nanoscale crystals are different from those of their bulk counterparts. ${ }^{[42,43]}$ Due to the small exciton binding energy in the bulk perovskite crystals, emissions of these materials are from radiative bimolecular recombination; the other two decay pathways, trap-assisted monomolecular recombination and Auger recombination, are non- 
radiative. ${ }^{[44]}$ Given that only bimolecular recombination is radiative, light output power of the devices is proportional to the bimolecular recombination rate, which can be expressed as

$$
P_{E L}=\beta B n^{2}(7)
$$

where $P_{E L}, \beta, B$, and $n$ are light output power, a constant, bimolecular recombination coefficient, and carrier density, respectively. Figure $4 \mathrm{a}$ and b show light output power-carrier density characteristics of the devices based on the PQD films annealed at $100{ }^{\circ} \mathrm{C}$ and $70{ }^{\circ} \mathrm{C}$, respectively. However, the experimental data (symbols) cannot be well fitted by Equation 7, and unexpectedly, light output powers are delivered under low carrier densities. This finding suggests that the PQDs undergo other radiative pathways in addition to the bimolecular recombination. Note that the PQDs are small enough to show the quantum confinement effect and their exciton binding energy $\left(E_{\mathrm{B}}\right)$ can be much larger than those of bulk materials. It is reported that perovskite nanoparticles can have a high exciton binding energy up to 300 meV. ${ }^{[42]}$ We can estimate the crossover carrier density $2 n_{e q}(T)$, above which excitons outnumber free electron-hole pairs, through the mass action law ${ }^{[45]}$ expressed by

$$
n_{e q}(T)=\left(\frac{m_{e} k_{B} T}{2 \pi \hbar^{2}}\right)^{\frac{3}{2}} e^{\frac{-E_{B}}{k_{B} T}}
$$

This equation forecasts that the crossover density for the PQDs with an exciton binding energy of $300 \mathrm{meV}$ is $\sim 10^{11} \mathrm{~cm}^{-3}$, above which a majority of excitons will spontaneously form. Thus, we believe that there is excitonic recombination - radiative monomolecular recombination in our PQDs. As shown in Figure 4a and b, the light output power-carrier density relation is better fitted under low excitation densities, by

$$
P_{E L}=\alpha k_{r} n(9),
$$

where $k_{r}$ and $\alpha$ are the radiative monomolecular recombination rate constant and a constant, respectively. This suggests that the light output power-carrier density relation can be regarded as the fact of excitonic recombination. 
To further prove this, we perform power-dependent PL decay studies of the PQD films as shown in Figure S7. Both of the films show biexponential decay characteristics, from which average lifetime constants $\left(\tau_{\text {ave }}\right)$ are calculated by using Equation S1 and S2 and summarized in Table S3. They show similar PL decays with little power dependence, depicting an unusual recombination behaviour compared to bulk $\mathrm{FAPbI}_{3}$ films. ${ }^{[46]}$ From the average decay time constants, we calculate the recombination rates of these PQDs. Figure 4c and d show the recombination rate-carrier density characteristics of both films, from which we estimate the monomolecular recombination rate constants and bimolecular recombination coefficients. Note that both PQD films show high monomolecular recombination rate constants of $\sim 10^{8} \mathrm{~s}^{-1}$, one order of magnitude higher than those of bulk crystals; The bimolecular recombination coefficients are $\sim 10^{-9}-10^{-8} \mathrm{~cm}^{3} \mathrm{~s}^{-1}$, of the same order of magnitude compared to those of the bulk crystals. ${ }^{[47]}$ In good agreement with the light output power-carrier density relation, the bimolecular recombination rate constants are at least one order of magnitude smaller than the monomolecular recombination rate constants under the carrier densities below $10^{15} \mathrm{~cm}^{-3}$, confirming the dominant excitonic recombination of the PQDs under low carrier densities.

For colloidal PQDs, the crystal size is not only relevant to the type and amount of ligands ${ }^{[26,48]}$, but also the reaction temperature ${ }^{[40,49]}$. Note that the nucleation and growth kinetics for colloidal CdTe, CdSe, and CdS nanocrystals are also time-dependent. ${ }^{[50]}$ Although it was reported that the nucleation and growth kinetics of PQDs are very fast and that the majority of growth occurs within the first $1-3 \mathrm{~s}^{[40]}$, the nucleation and growth could become slower in our solid-state synthesis. Thus, it would be natural to consider whether the in-situ grown PQDs have the time-dependent growth feature. Interestingly, as shown in Figure S8, the in-situ grown PQDs show enhanced crystallinity with longer reaction time, and consequently red shifts of the PL spectra. Specifically, the diffraction intensity increases with longer annealing time (Figure S8a), implying higher crystallinity. This agrees with an increased crystal size from $6.9 \mathrm{~nm}$ to 
$10.4 \mathrm{~nm}$. The corresponding PL spectra in Figure S8b also show red shifts peaking from 690 $\mathrm{nm}$ to $760 \mathrm{~nm}$ with extended annealing time.

These PQDs are also employed in LEDs with the same device architecture mentioned above. Figure 5 shows device performance of these devices. Notably, the devices based on the PQDs with a longer annealing time show higher current density due to higher crystallinity and consequently better charge-transport properties. The turn-on voltages of these devices gradually decrease with longer annealing time (Figure 5a), which can be explained by the reduced energy bandgaps of the PQDs. In addition, the PQDs with longer annealing time deliver better device performance. The device based on the PQD film annealed for 120 min gives a high radiance of $20.9 \mathrm{~W} \mathrm{sr}^{-1} \mathrm{~m}^{-2}$ at $3.6 \mathrm{~V}$ and a high EQE of $4.3 \%$ at $2.4 \mathrm{~V}$ (corresponding to a current density of $32.6 \mathrm{~mA} \mathrm{~cm}^{-2}$ ) (Figure 5 and Table 2). More importantly, as described in Figure 5d and Table 2, the EL spectra of these devices also exhibit red shifts ranging from $690 \mathrm{~nm}$ to $762 \mathrm{~nm}$ as extending the thermal annealing time. These results further demonstrate that the in-situ generated PQDs have tunable PL and EL emissions comparable to the colloidal QDs, while with much simpler synthesis process, highlighting their great potential in applications regarding thin films of colloidal PQDs.

In summary, we have demonstrated a straightforward, in-situ route to produce PQDs for efficient LEDs based on precursor solution and annealing processing modulation. Similar to epitaxial- and colloidal-grown PQDs, these $\mathrm{FAPbI}_{3}$-based in-situ grown PQDs exhibit the sizedependent quantum confinement effect and consequently tunable emissions. The emission spectral peak of the present in-situ grown PQDs can be tuned between $667 \mathrm{~nm}$ and $778 \mathrm{~nm}$ by changing the annealing temperature from $50{ }^{\circ} \mathrm{C}$ to $100{ }^{\circ} \mathrm{C}$ (for $10 \mathrm{~min}$ ). By extending the annealing time at $70{ }^{\circ} \mathrm{C}$ from 10 to $120 \mathrm{~min}$, the spectral peak change from $690 \mathrm{~nm}$ to $760 \mathrm{~nm}$. The narrow-bandwidth emission and its high color stability in highly heterogeneous samples are provided by the efficient energy transfer between nanostructures that sweeps away the 
disorder effects and bandgap energy variations. Notably, the in-situ grown $\mathrm{FAPbI}_{3} \mathrm{PQDs}_{\mathrm{b}}$ based devices deliver high EQEs up to $11.0 \%$ in LEDs, indicating great potential of the reported preparation method and material system for cheap, efficient and color-tunable LEDs. Interestingly, these in-situ generated PQDs have emission from radiative monomolecular recombination, promoting the device efficiencies under low excitation densities. Our study opens up a new route for robust synthesis of PQD-based films for high-performance optoelectronic devices.

\section{Supporting Information}

Supporting Information is available from the Wiley Online Library or from the author.

\section{Acknowledgements}

This work is supported by the ERC Starting Grant (717026), the Carl Tryggers Stiftelse, the European Commission Marie Skłodowska-Curie Actions (691210), and the Swedish Government Strategic Research Area in Materials Science on Functional Materials at Linköping University (Faculty Grant SFO-Mat-LiU no. 2009-00971). H. Y., H. W., Z. Y. and J. Z. acknowledge the financial support of the China Scholarship Council. X. K. L. is a VINNMER and Marie Skłodowska-Curie Fellow. A. A. B. is a Royal Society University Research Fellow.

\section{Reference}

[1] A. P. Alivisatos, Science 1996, 271, 933.

[2] Q. Sun, Y. A. Wang, L. S. Li, D. Wang, T. Zhu, J. Xu, C. Yang, Y. Li, Nat. Photonics 2007, 1, 717.

[3] P. V. Kamat, J. Phys. Chem. C 2008, 112, 18737.

[4] X. Lin, X. Dai, C. Pu, Y. Deng, Y. Niu, L. Tong, W. Fang, Y. Jin, X. Peng, Nat. Commun. 2017, 8, 1132.

[5] T. C. Harman, P. J. Taylor, M. P. Walsh, B. E. La Forge, Science 2002, 297, 2229.

[6] C. R. Kagan, E. Lifshitz, E. H. Sargent, D. V. Talapin, Science 2016, 353, 5523.

[7] Y. Shirasaki, G. J. Supran, M. G. Bawendi, V. Bulović, Nat. Photonics 2013, 7, 13.

[8] V. L. Colvin, M. C. Schlamp, A. P. Alivisatos, Nature 1994, 370, 354.

[9] X. Dai, Z. Zhang, Y. Jin, Y. Niu, H. Cao, X. Liang, L. Chen, J. Wang, X. Peng, Nature 2014, 515, 96.

[10] X. Dai, Y. Deng, X. Peng, Y. Jin, Adv. Mater. 2017, 29, 1607022.

[11] J.-P. Correa-Baena, M. Saliba, T. Buonassisi, M. Grätzel, A. Abate, W. Tress, A. Hagfeldt, Science 2017, 358, 739.

[12] S. A. Veldhuis, P. P. Boix, N. Yantara, M. J. Li, T. C. Sum, N. Mathews, S. G. Mhaisalkar, Adv. Mater. 2016, 28, 6804. 
[13] Z.-K. Tan, R. S. Moghaddam, M. L. Lai, P. Docampo, R. Higler, F. Deschler, M. Price, A. Sadhanala, L. M. Pazos, D. Credgington, F. Hanusch, T. Bein, H. J. Snaith, R. H. Friend, Nat. Nanotechnol. 2014, 9, 687.

[14] H. Cho, S. H. Jeong, M. H. Park, Y. H. Kim, C. Wolf, C. L. Lee, J. H. Heo, A. Sadhanala, N. S. Myoung, S. Yoo, S. H. Im, R. H. Friend, T. W. Lee, Science 2015, 350, 1222.

[15] S. Ahn, M.-H. Park, S.-H. Jeong, Y.-H. Kim, J. Park, S. Kim, H. Kim, H. Cho, C. Wolf, M. Pei, H. Yang, T.-W. Lee. Adv. Funct Mater. 2018, 1807535.

[16] Y.-H. Kim, S. Kim, S. H. Jo, T.-W. Lee, Small Methods. 2018, 2, 1800093.

[17] N. Wang, L. Cheng, R. Ge, S. Zhang, Y. Miao, W. Zou, C. Yi, Y. Sun, Y. Cao, R. Yang, Y. Wei, Q. Guo, Y. Ke, M. Yu, Y. Jin, Y. Liu, Q. Ding, D. Di, L. Yang, G. Xing, H. Tian, C. Jin, F. Gao, R. H. Friend, J. Wang, W. Huang, Nat. Photonics 2016, 10, 699.

[18] B. Zhao, S. Bai, V. Kim, R.Lamboll, R. Shivanna, F. Auras, J. M. Richter, L. Yang, L. Dai, M. Alsari, X.-J. She, Lu. Liang, J. Zhang, S. Lilliu, P. Gao, H. J. Snaith, J. Wang, N. C. Greenham, R. H. Friend, D. Di, Nat. Photonics 2016, Preprint at https://arxiv.org/abs/1804.09785.

[19] K. Lin, J. Xing, L. Quan, F. P. G.de Arquer, X. Gong, J. Lu, L. Xie, W. Zhao, D. Zhang, C. Yan, W. Li, X. Liu, Y. Lu, J. Kirman, E. H. Sargent, Q. Xiong, Z. Wei, Nature 2018, 562, 245.

[20] Y. Cao, N. Wang, H. Tian, J. Guo, Y. Wei, H. Chen, Y. Miao, W. Zou, K. Pan, Y. He, H. Cao, Y. Ke, M. Xu, Y. Wang, M. Yang, K. Du, Z. Fu, D. Kong, D. Dai, Y. Jin, G. Li, H. Li, Q. Peng, J. Wang, W. Huang, Nature 2018, 562, 249.

[21] T. Chiba, Y. Hayashi, H. Ebe, K. Hoshi, J. Sato, S. Sato, Y.-J. Pu, S. Ohisa, J. Kido, Nat. Photonics 2018, 12, 681.

[22] X. Li, Y. Wu, S. Zhang, B. Cai, Y. Gu, J. Song, H. Zeng, Adv. Funct. Mater. 2016, 26, 2435.

[23] T. Chiba, K. Hoshi, Y.-J. Pu, Y. Takeda, Y. Hayashi, S. Ohisa, S. Kawata, J. Kido, ACS Appl. Mater. Interfaces 2017, 9, 18054.

[24] J. Song, J. Li, L. Xu, J. Li, F. Zhang, B. Han, Q. Shan, H. Zeng, Adv. Mater. 2018, 30, 1800764.

[25] J. Song, J. Li, X. Li, L. Xu, Y. Dong, H. Zeng, Adv. Mater. 2015, 27, 7162.

[26] L. C. Schmidt, A. Pertegás, S. González-Carrero, O. Malinkiewicz, S. Agouram, G. Mínguez Espallargas, H. J. Bolink, R. E. Galian, J. Pérez-Prieto, J. Am. Chem. Soc. 2014, 136, 850.

[27] J. Li, L. Xu, T. Wang, J. Song, J. Chen, J. Xue, Y. Dong, B. Cai, Q. Shan, B. Han, H. Zeng, Adv. Mater. 2017, 29, 1603885.

[28] N. J. Jeon, J. H. Noh, W. S. Yang, Y. C. Kim, S. Ryu, J. Seo, S. Il Seok, Nature 2015, $517,476$.

[29] U. Holzwarth, N. Gibson, Nat. Nanotechnol. 2011, 6, 534.

[30] F. Ma, J. Li, W. Li, N. Lin, L. Wang, J. Qiao, Chem. Sci. 2017, 8, 800.

[31] C. C. Stoumpos, C. D. Malliakas, M. G. Kanatzidis, Inorg. Chem. 2013, 52, 9019.

[32] L. Brus, J. Phys. Chem. 1986, 90, 2555.

[33] D. S. Dolzhnikov, C. Wang, Y. Xu, M. G. Kanatzidis, E. A. Weiss, Chem. Mater. 2017, 29, 7901.

[34] N. Pellet, P. Gao, G. Gregori, T.-Y. Yang, M. K. Nazeeruddin, J. Maier, M. Grätzel, Angew. Chem. Int. Ed. 2014, 53, 3151.

[35] K. Galkowski, A. Mitioglu, A. Miyata, P. Plochocka, O. Portugall, G. E. Eperon, J. T.W. Wang, T. Stergiopoulos, S. D. Stranks, H. J. Snaith, R. J. Nicholas, Energy Environ. Sci. 2016, 9, 962. 
[36] L. N. Quan, Y. Zhao, F. P. G. Arquer, R. Sabatini, G. Walters, O. Voznyy, R. Comin, Y. Li, J. Z. Fan, H. Tan, J. Pan, M. Yuan, O. M. Bakr, Z. Lu, D. Ha Kim, E. H. Sargent, Nano Lett. 2017, 17, 3701.

[37] A. Swarnkar, R. Chulliyil, V. K. Ravi, M. Irfanullah, A. Chowdhury, A. Nag, Angew. Chem. 2015, 127, 15644.

[38] M. B. Price, J. Butkus, T. C. Jellicoe, A. Sadhanala, A. Briane, J. E. Halpert, K. Broch, J. M. Hodgkiss, R. H. Friend, F. Deschler, Nat. Commun. 2015, 6, 8420.

[39] H. Wang, H. Yu, W. Xu, Z. Yuan, Z. Yan, C. Wang, X. Liu, M. Fahlman, J.-M. Liu, X.K. Liu, F. Gao, J. Mater. Chem. C, 2018, 6, 6996.

[40] L. Protesescu, S. Yakunin, M. I. Bodnarchuk, F. Krieg, R. Caputo, C. H. Hendon, R. X. Yang, A. Walsh, M. V. Kovalenko, Nano Lett. 2015, 15, 3692.

[41] E. T. Hoke, D. J. Slotcavage, E. R. Dohner, A. R. Bowring, H. I. Karunadasa, M. D. McGehee, Chem Sci 2015, 6, 613.

[42] K. Zheng, Q. Zhu, M. Abdellah, M. E. Messing, W. Zhang, A. Generalov, Y. Niu, L. Ribaud, S. E. Canton, T. Pullerits, J. Phys. Chem. Lett. 2015, 6, 2969.

[43] H.-H. Fang, L. Protesescu, D. M. Balazs, S. Adjokatse, M. V. Kovalenko, M. A. Loi, Small 2017, 13, 1700673.

[44] M. B. Johnston, L. M. Herz, Acc. Chem. Res. 2016, 49, 146.

[45] V. Sarritzu, N. Sestu, D. Marongiu, X. Chang, Q. Wang, M. A. Loi, F. Quochi, M. Saba, A. Mura, G. Bongiovanni, Adv. Opt. Mater. 2017, 1700839, 6.

[46] G. Xing, B. Wu, X. Wu, M. Li, B. Du, Q. Wei, J. Guo, E. K. L. Yeow, T. C. Sum, W. Huang, Nat. Commun. 2017, 8, 14558.

[47] V. D’Innocenzo, A. R. Srimath Kandada, M. De Bastiani, M. Gandini, A. Petrozza, J. Am. Chem. Soc. 2014, 136, 17730.

[48] S. G. Carrero, R. E. Galian, J. P. Prieto, J. Mater. Chem. A, 2015, 3, 9187.

[49] H. Huang, A. S. Susha, S. V. Kershaw, T. F. Hung, A. L. Rogach, Adv. Sci. 2015, 2, 1500194.

[50] Z. A. Peng, X. Peng, J. Am. Chem. Soc. 2001, 123, 183. 
(a)
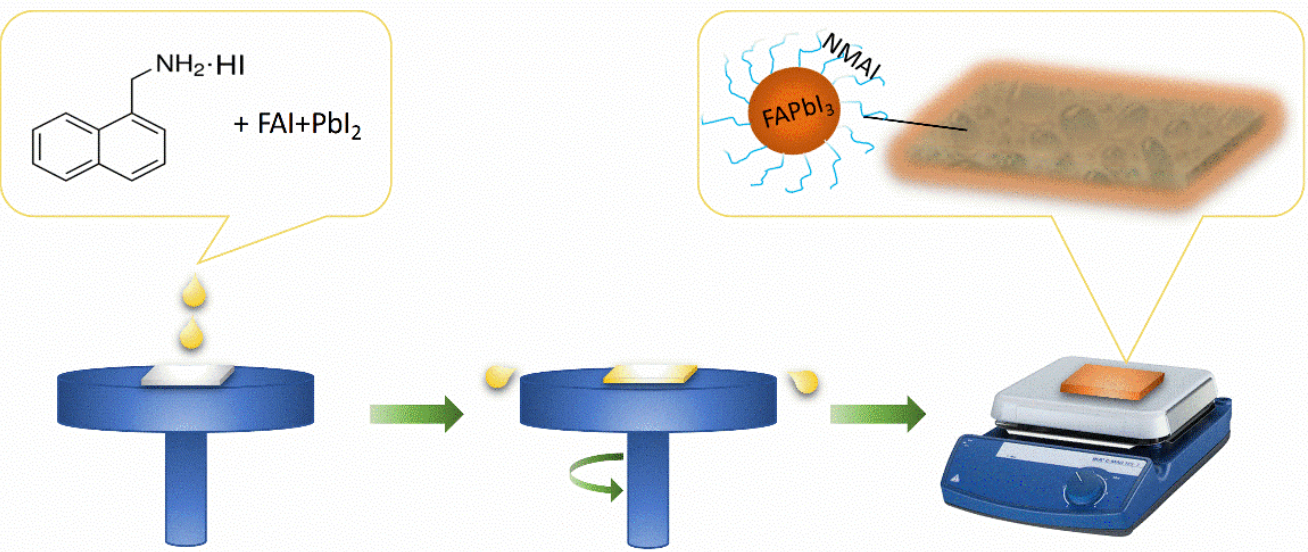

Spin-coating

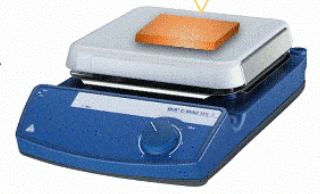

Annealing

(b)

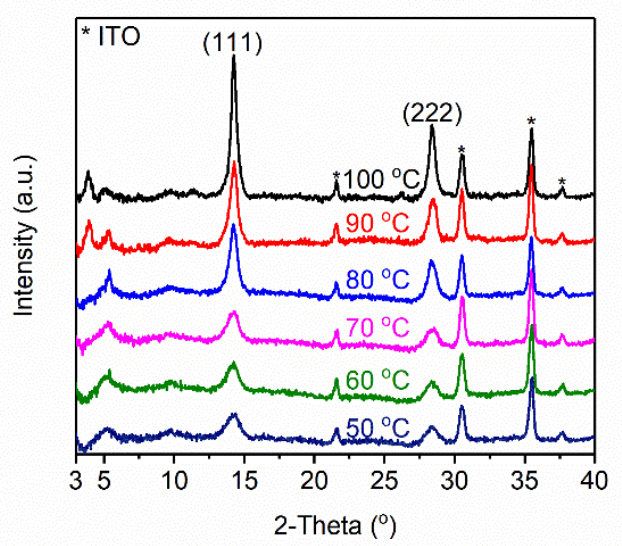

(d)

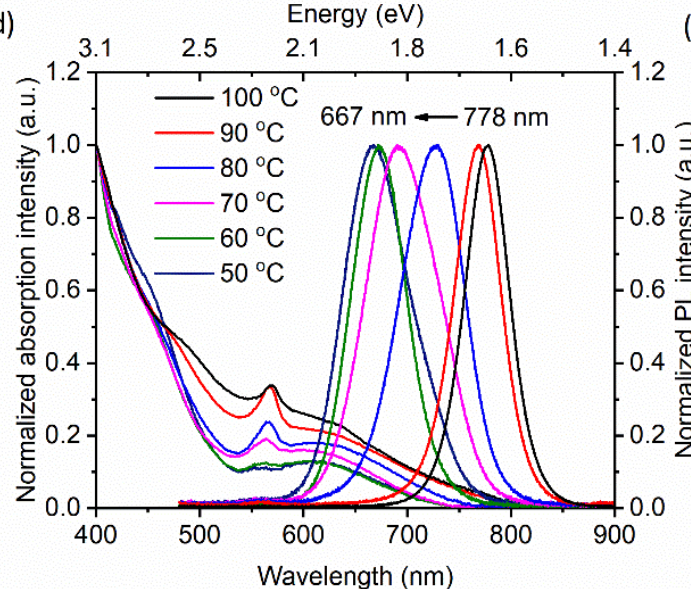

(c)

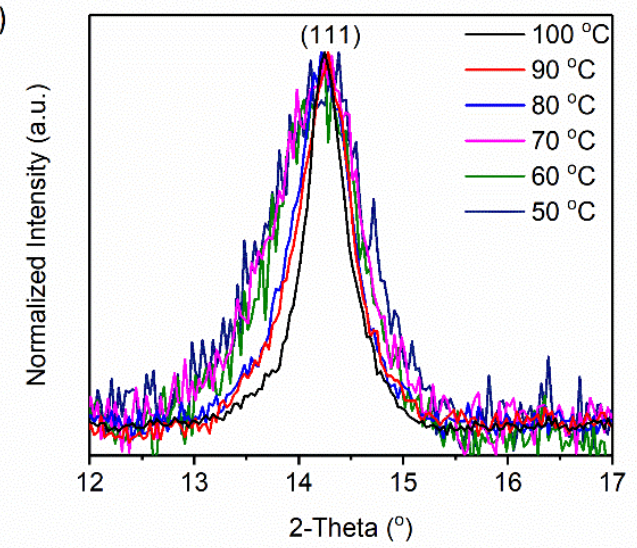

(e)

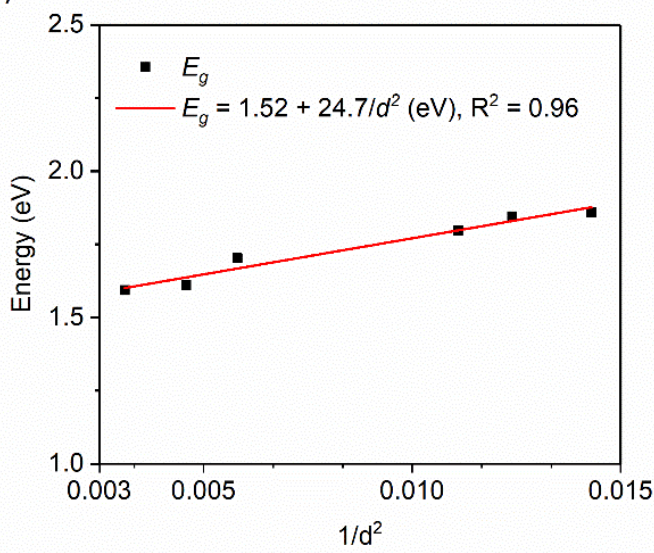

Figure 1. (a) Schematic diagram for in-situ synthesis of PQDs. (b) XRD patterns, (c) normalized diffraction intensity of the (111) lattice plane, and (d) normalized UV-Vis absorption and PL spectra of in-situ synthesized $\mathrm{FAPbI}_{3} \mathrm{PQD}$-based films annealed at various temperatures. (e) Relation between energy bandgaps and average diameters of the in-situ synthesized PQDs, which can be well fitted by the equation of $E_{\mathrm{g}}=1.52+24.7 / d^{2}(\mathrm{eV})$, where $d$ is the crystal size in $\mathrm{nm}$. 

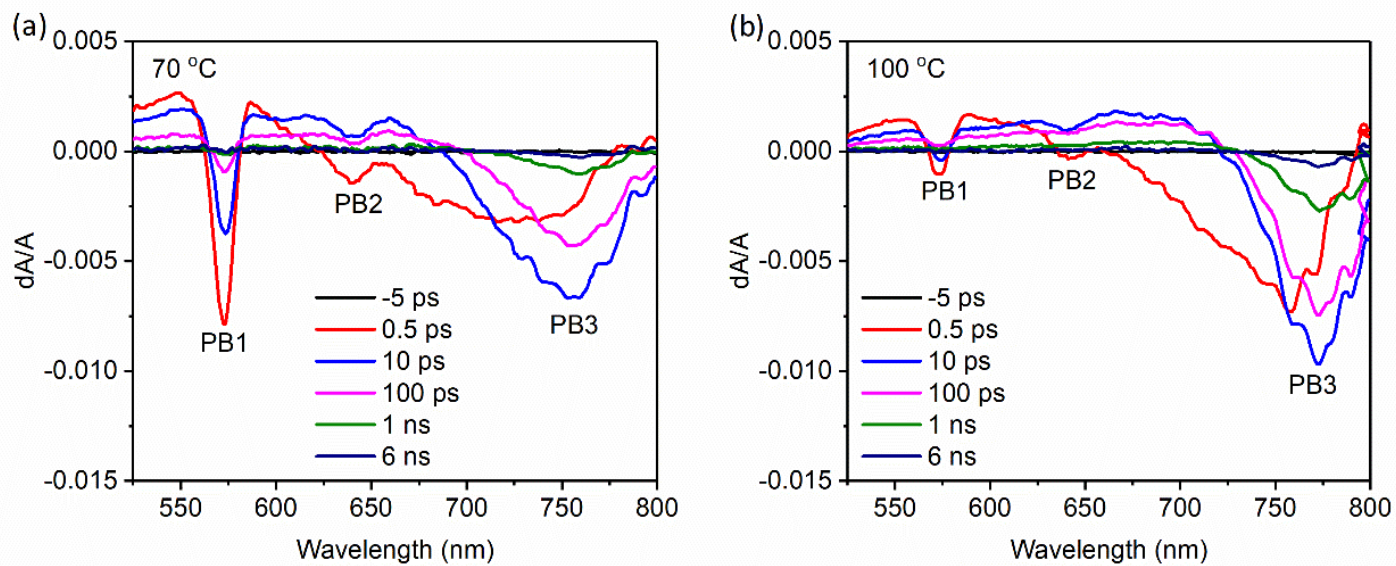

Figure 2. Transient absorption spectra of the in-situ synthesized PQD films annealed at (a) 70 ${ }^{\circ} \mathrm{C}$ and (b) $100{ }^{\circ} \mathrm{C}$, respectively, for $10 \mathrm{~min}$. The films were excited at $500 \mathrm{~nm}$.

(a)
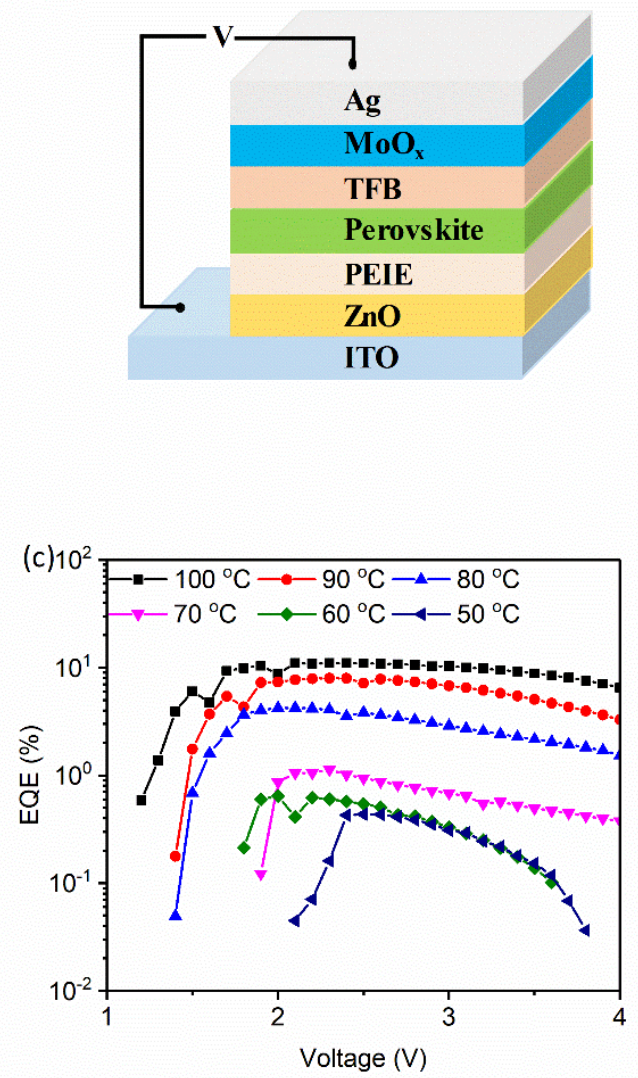

(b)

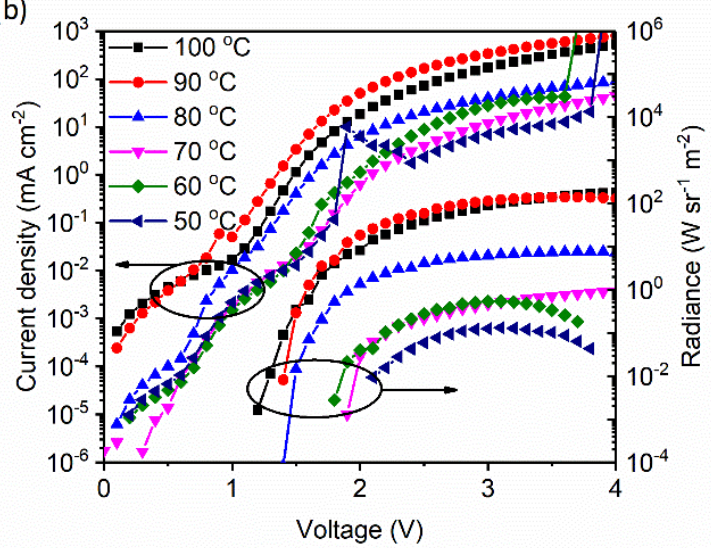

(d)

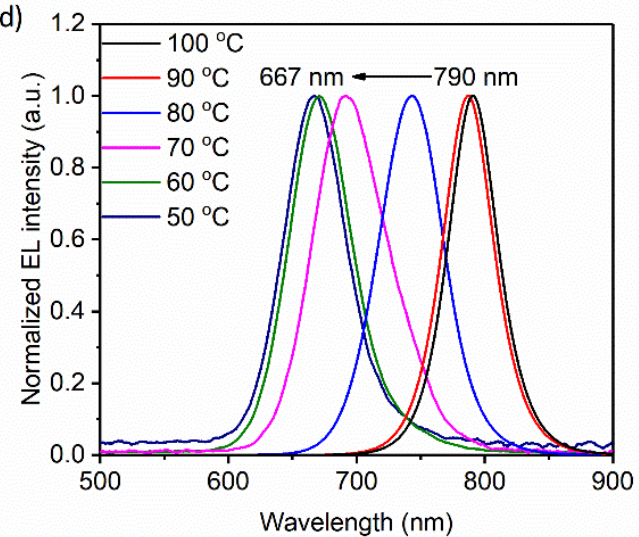

Figure 3. Devices based on in-situ synthesized $\mathrm{FAPbI}_{3} \mathrm{PQD}$ films annealed at various temperatures for 10 min. (a) Schematic diagram of the device architecture. (b) Current densityvoltage-radiance, (c) EQE-voltage characteristics, and (d) normalized EL spectra. 
Table 1. Characteristics of LEDs based on in-situ synthesized $\mathrm{FAPbI}_{3} \mathrm{PQDs}$ annealed at various temperatures for $10 \mathrm{~min}$.

\begin{tabular}{cccccc}
\hline $\mathrm{T}\left({ }^{\circ} \mathrm{C}\right)$ & $\lambda_{\mathrm{EL}}(\mathrm{nm})$ & $\mathrm{V}_{\text {on }}(\mathrm{V})$ & $\mathrm{L}\left(\mathrm{cd} \mathrm{m}^{-2}\right)$ & $\mathrm{EQE}(\%)$ & $\mathrm{R}\left(\mathrm{W} \mathrm{sr}^{-1} \mathrm{~m}^{-2}\right)$ \\
\hline 100 & 790 & 1.2 & 55.1 & 11.0 & 178.4 \\
90 & 787 & 1.4 & 43.6 & 8.0 & 141.1 \\
80 & 743 & 1.4 & 11.2 & 4.2 & 7.7 \\
70 & 691 & 1.9 & 22.1 & 1.1 & 1.0 \\
60 & 671 & 1.8 & 22.3 & 0.6 & 0.5 \\
50 & 667 & 2.0 & 5.9 & 0.4 & 0.1 \\
\hline
\end{tabular}
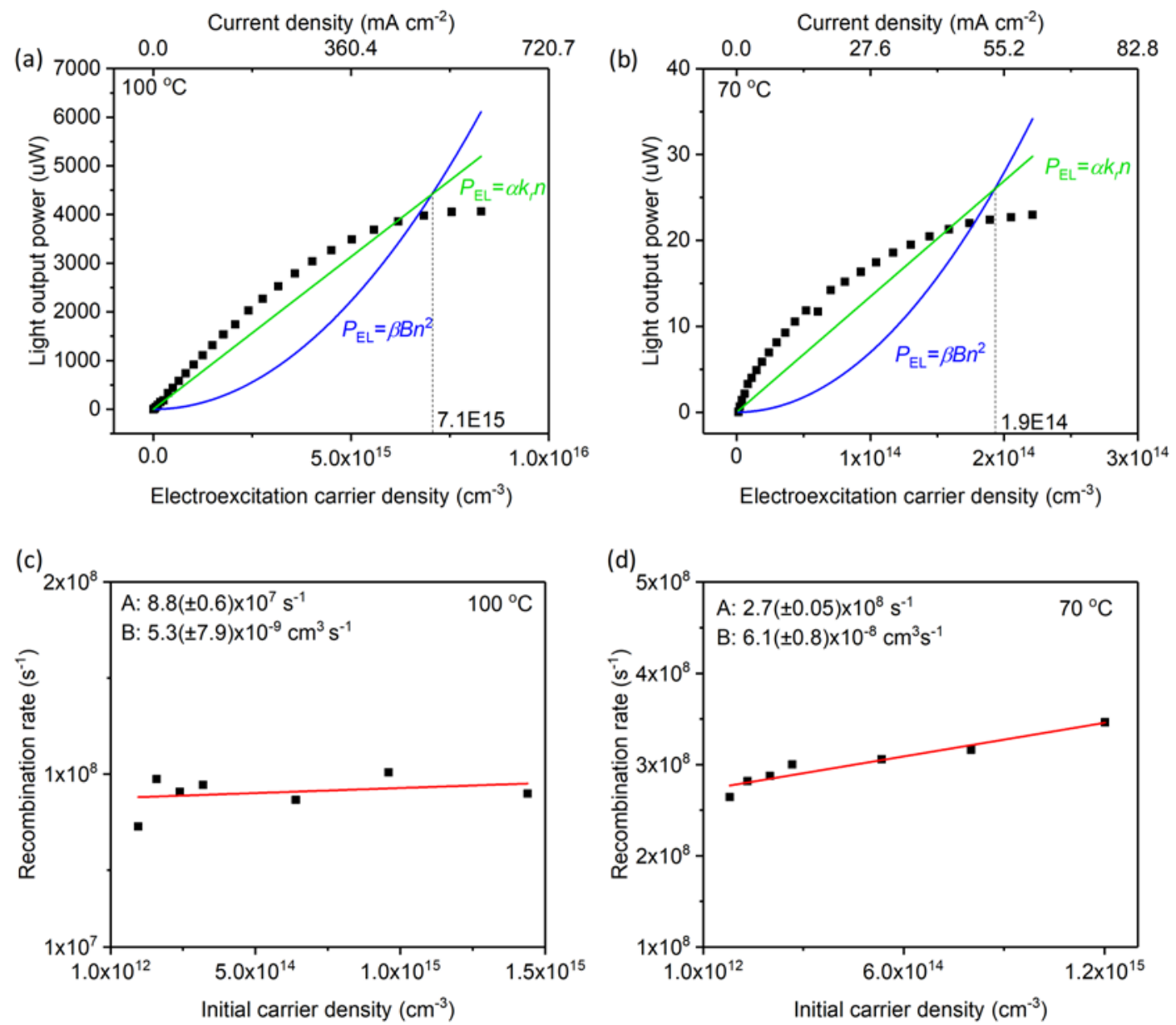

Figure 4. Carrier dynamics of the in-situ synthesized PQD films annealed at $100{ }^{\circ} \mathrm{C}$ and $70{ }^{\circ} \mathrm{C}$, respectively. (a), (b) Light output power-electroexcitation carrier density characteristics of their devices. (c), (d) Recombination rate-carrier density characteristics. The monomolecular recombination rate constant and bimolecular recombination coefficient are estimated by fitting the data using $k_{\text {total }}=A+B n_{P L}$, where $k_{\text {total }}$ is the total recombination rate calculated from the average PL lifetime constants. 

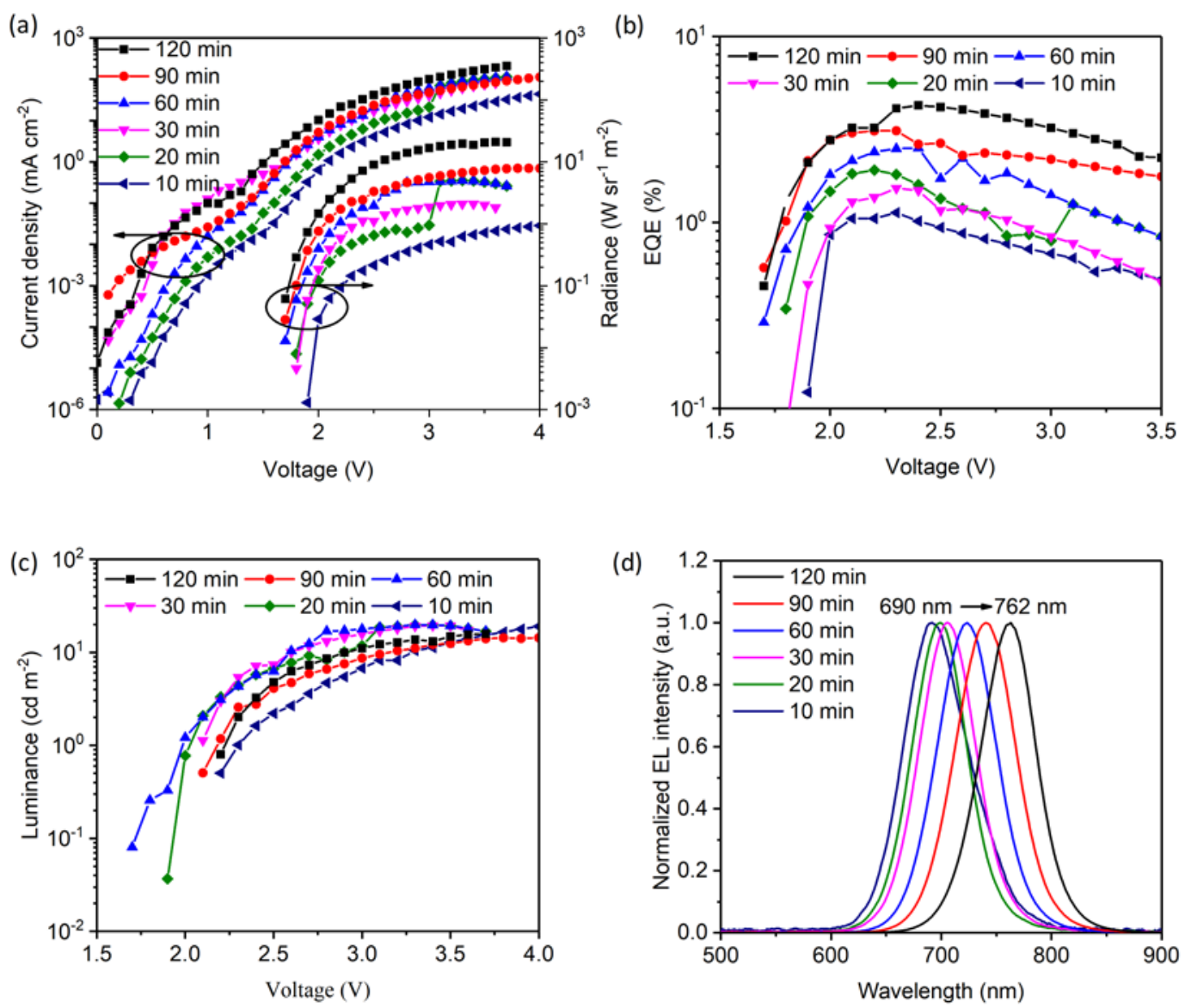

Figure 5. Device performance of the LEDs based on in-situ synthesized $\mathrm{FAPbI}_{3} \mathrm{PQDs}$ annealed at $70{ }^{\circ} \mathrm{C}$ for various times. (a) Current density-voltage-radiance, (b) EQE-voltage and (c) luminance-voltage plots, and (d) normalized EL spectra.

Table 2. Characteristics of LEDs based on in-situ synthesized FAPbI 3 PQDs annealed at 70 ${ }^{\circ} \mathrm{C}$ for various times.

\begin{tabular}{|c|c|c|c|c|c|}
\hline$T(\min )$ & $\lambda_{\mathrm{EL}}(\mathrm{nm})$ & $\mathrm{V}_{\text {on }}(\mathrm{V})$ & $L\left(\mathrm{~cd} \mathrm{~m}^{-2}\right)$ & EQE (\%) & $R\left(W s^{-1} m^{-2}\right)$ \\
\hline 120 & 762 & 1.7 & 15.9 & 4.3 & 20.9 \\
\hline 90 & 741 & 1.7 & 14.4 & 3.1 & 7.9 \\
\hline 60 & 724 & 1.7 & 19.8 & 2.5 & 5.0 \\
\hline 30 & 706 & 1.8 & 19.4 & 1.5 & 2.1 \\
\hline 20 & 699 & 1.8 & 19.8 & 1.9 & 5.0 \\
\hline 10 & 690 & 1.9 & 22.1 & 1.1 & 1.0 \\
\hline
\end{tabular}

